\title{
Pace of leprosy elimination and support teams in Bihar State, India
}

\author{
P. VIJAYAKUMARAN, T. PRABHAKAR RAO \& \\ P. KRISHNAMURTHY \\ Damien Foundation India Trust, 27 Venugopal Avenue, \\ Spur Tank Road, Chetpet, Chennai, India
}

Accepted for publication 15 September 1999

\begin{abstract}
Summary Despite the extensive implementation of multiple drug therapy (MDT) in most leprosy-endemic countries world-wide since 1982, bringing about a remarkable reduction in prevalence, there are still regions at the sub-national level where the implementation of MDT remains difficult. The state of Bihar (population 86.3 million) in India is a good example of such a region. Previously rated as one of the most highly endemic states, it still contributes about $21 \%$ of the total caseload in India and about $12 \%$ of the global caseload. For various reasons, case-finding and drug treatment have lagged behind the progress made in most other states in the country and in 1996, the Damien Foundation India Trust (DFIT) volunteered technical support to increase the pace of elimination. Sixteen out of the 39 districts in the state were allocated, with a population of 41.8 million. Support teams, including a Medical Advisor and a Non-Medical Supervisor, both with over 10 years experience of leprosy work and control programmes, were provided to assist and work alongside government staff in case detection, treatment delivery, case-holding and discharge in their respective areas of operation. New case detection by intensive survey increased by $394 \%$ and total new case detection by $226 \%$ during the year 1996-1997, with similar trends in the following year. Striking improvements were also observed in MDT coverage, treatment regularity, monitoring and discharge of patients and in the training of local staff. This collaboration between a non-government agency (DFIT) and the staff of the National Leprosy Eradication Programme in 16 out of 39 districts in the State of Bihar has clearly been extremely successful. Similar approaches in the remaining districts of Bihar, and in other parts of India, where the infrastructure is available but inadequate, may contribute significantly to achieving the elimination goal at national and sub-national levels.
\end{abstract}

\section{Introduction}

With the introduction of multiple drug therapy (MDT) in the 1980s, the prevalence of leprosy (active caseload) has been significantly reduced in many parts of the world. There is a possibility of reducing prevalence to the elimination level of less than 1 case per 10,000 of the 
population, as defined by the World Health Organisation (WHO), but a number of countries still have several leprosy-endemic pockets with inadequate MDT coverage for various reasons, calling for urgent attention.

India is endemic for leprosy. Although MDT programmes have been introduced in the majority of leprosy endemic regions in the country since 1983, India still contributed about $70 \%$ of the global leprosy caseload during 1996. The trend continued even in 1998. ${ }^{1,2}$ Many of the leprosy endemic countries showed a decline in new case detection rates following implementation of an intensive MDT programme, but there has been no such decline observed in India. ${ }^{3}$

There are a few leprosy-endemic regions where an intensive MDT programme could not be carried out, and Bihar State in India is one such region, MDT having been implemented only recently. We present a description of a strategy adopted in this region for the successful implementation of an MDT programme during 1996-1998, based on the provision of support teams of experienced workers by the Damien Foundation India Trust (DFIT).

\section{Materials and methods}

\section{BACKGROUND INFORMATION}

Bihar State is situated in northern India, bordering Nepal. This state is rich in mineral deposits and water resources for agriculture. Bihar State, with a population of 86.3 million (Table 1), has been one of the major contributors to the leprosy caseload in India and world-wide. It was observed that Bihar contributed about $21 \%$ of the caseload in India and $12 \%$ of the global caseload (1996). There were 127,000 leprosy patients on record in 1996. There was patchy coverage with MDT and the annual new case detection fluctuated widely (Table 2) from 1991 to 1996 , indicating spurts of leprosy control activities.

The Government of India provided support to the state governments for implementation of National Leprosy Eradication Programme (NLEP), but Bihar had a range of problems that delayed implementation until 1993. Backlog case records were screened and removed in a procedure called 'cleaning of records'. This procedure was carried out twice (1993 and 1996), but in spite of all these efforts, only $50 \%$ of the patients on record were on MDT even during early 1996.

Some of the problems are listed here.

- Staffing for the NLEP was inadequate, though there was provision for recruitment of staff.

- Supervision of field staff and technical support was grossly deficient.

- There was persistent mismatch between the work culture and programme expectation.

Table 1. Profile of Bihar state

\begin{tabular}{lc}
\hline & \\
Population & $86 \cdot 3$ million \\
Number of revenue districts & 39 \\
Population density per $\mathrm{km}^{2}$ & $497(257)^{*}$ \\
Population below poverty line & $40 \%(29 \%)^{*}$ \\
Literacy rate & $30 \%(52 \%)^{*}$ \\
\hline
\end{tabular}

*National average. 
Table 2. Leprosy profile-trend in Bihar

\begin{tabular}{lccccc}
\hline Item & 1991 & 1992 & 19993 & 1994 & 1995 \\
\hline Leprosy patients on record & 451,357 & 352,193 & 202,582 & 180,582 & 149,572 \\
\% of leprosy patients on MDT & $9 \cdot 8$ & $7 \cdot 3$ & $22 \cdot 1$ & $34 \cdot 0$ & $49 \cdot 8$ \\
New leprosy patients detected & 31,481 & 34,376 & 86,281 & 62,992 & 48,004 \\
Prevalence of leprosy per 10,000 & $52 \cdot 2$ & $40 \cdot 8$ & $23 \cdot 5$ & $20 \cdot 9$ & $17 \cdot 3$ \\
\hline
\end{tabular}

- Most of the leprosy staff were not trained or had been trained 20 years ago.

- Supply of MDT drugs was erratic and inadequate.

- Mobility of the existing staff was poor. There was a lack of vehicles and the existing vehicles were being used for other purposes. Public transport in this region was rudimentary. Road communications were very poor.

- The region is prone to natural disasters, especially floods, and bandits are common. Hence about $30-40 \%$ of the villages become inaccessible for about 4-6 months.

- Extreme weather conditions are common. The temperature sores to $45^{\circ} \mathrm{C}$ during summer and drops to $4-6^{\circ} \mathrm{C}$ during winter.

The Damien Foundation India Trust (DFIT) came forward to assist in implementation of NLEP in Bihar in 1995. Out of 39 districts in the state, 16 were allotted for assistance from DFIT in February 1996. These 16 districts had a population of 41.8 million (43.9\% of the population in the state). The leprosy caseload in these districts $(52,388)$ was $50 \%$ of the total caseload in the state. Each of the districts was provided with a support team consisting of one District Leprosy Advisor (DLA), one Non-Medical Supervisor (NMS) and a driver with a jeep. In some of the larger districts, two or three NMS were placed under the District Leprosy Advisor. The DLA and NMS had more than 10 years experience in implementation of MDT. They were mostly from other states in India where an MDT programme had already been successfully implemented. The support team was expected to assist programme managers at the district and peripheral leprosy units to plan, implement and monitor the MDT programme.

\section{Job responsibilities of support team}

- To consolidate baseline data.

- To assist in planning and implement case detection and treatment delivery.

- To ensure regular treatment delivery.

- To ensure timely case discharge (release from treatment-RFT).

- To train programme personnel (leprosy and primary health care).

- To guide in preparation of report.

- To guide programme manager to monitor the programme activities.

They also liaised between state level programme managers and district level managers. This was achieved through monthly meetings of support teams with the State Leprosy Officer. They offered guidance in the utilization of MDT funds as per the NLEP guidelines. The support teams were co-ordinated by two senior level Medical Advisors from DFIT who were stationed in Bihar State. 


\section{Results}

- All the supported districts prepared the plan and implemented treatment delivery.

- When the programme staff were inadequate, the available staff were redistributed to cover the population.

- New case detection activities were planned and implemented.

- Work output was monitored through periodic review meetings, reports and during field visits.

- Technical support was given in training of staff (Table 3), screening of population, organising special screening programmes, ${ }^{4,5}$ management of complications and drug management.

The performance of NLEP in Bihar during previous years (1991-1996) is shown in Table 2. The performance during April 1995 to March 1996 is taken as the baseline for comparison. In the 16 DFIT-assisted districts:

- New case detection by survey increased by $394 \%$ and total new case detection was $226 \%$ more during the year 1996-1997 (Figure 1). A similar trend was also observed during the next year.

- Treatment completion rate would have been a more accurate indicator, but it was difficult to obtain. Hence, we used the proportion of leprosy patients removed from the registers by completion of treatment, i.e. RFT, which was an indirect indicator of treatment completion. During previous years, about $50 \%$ of leprosy patients were discharged by modes other than treatment completion, e.g. migration, not traceable, died, indicating that treatment completion was inadequate. With technical assistance from the support team, the case discharge increased by $160 \%$ and discharge by treatment completion by $230 \%$ (Figure 2) during subsequent years.

- MDT coverage of cases on record improved to $100 \%$ and treatment regularity improved from $49 \%$ to $82 \%$ (Figure 3 ).

Table 3. Personnel trained by DFIT in Bihar (1996-1998)

\begin{tabular}{lr}
\hline Category of personnel & $*$ Number trained \\
\hline 1. PMW \& NMS & 2655 \\
2. Medical Officer-NLEP & 51 \\
3. Medical Officer-PHC & 643 \\
4. PHC workers & 270 \\
5. Medical Practitioners & 269 \\
6. Others & 740 \\
Total & 4628 \\
\hline
\end{tabular}

\footnotetext{
*Includes personnel from DFIT assisted and other districts of Bihar.

DFIT $=$ Damien Foundation India Trust

PMW $=$ Paramedical Worker (leprosy)

NMS $=$ Non-Medical Supervisor

NLEP $=$ National Leprosy Eradication Programme

PHC $=$ Primary Health Centre
} 


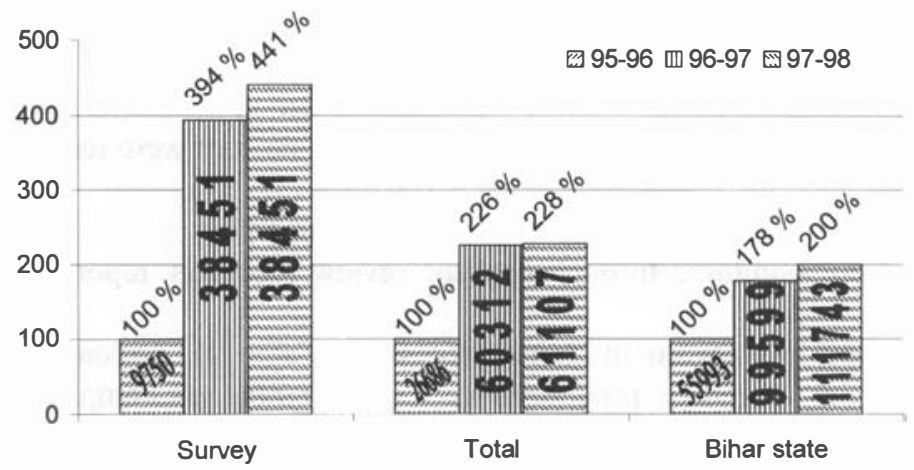

Figure 1. New case detection 1995-1998. Improvement (\%) in DFIT-assisted districts and whole of Bihar State.

- The treatment delivery programme had become fairly regular. Areas that were likely to become inaccessible were identified. Leprosy patients in these areas were provided with 2-3 months supply of MDT just prior to the start of the rainy season, to ensure uninterrupted treatment.

- Alternate arrangements were made for treatment delivery during difficult situations.

- Large numbers of staff were trained not only from DFIT-assisted districts but from other districts too.

- The District Leprosy Society (DLS) was activated and co-operation was obtained from the district level health authority and administrative authority. The DLS started releasing MDT funds to implement various programme activities.

- Vehicles were released for programme activities. New vehicles were obtained from the MDT funds wherever needed.

- An adequate stock of MDT drugs was obtained from Government of India and the same was made available to the peripheral leprosy units.

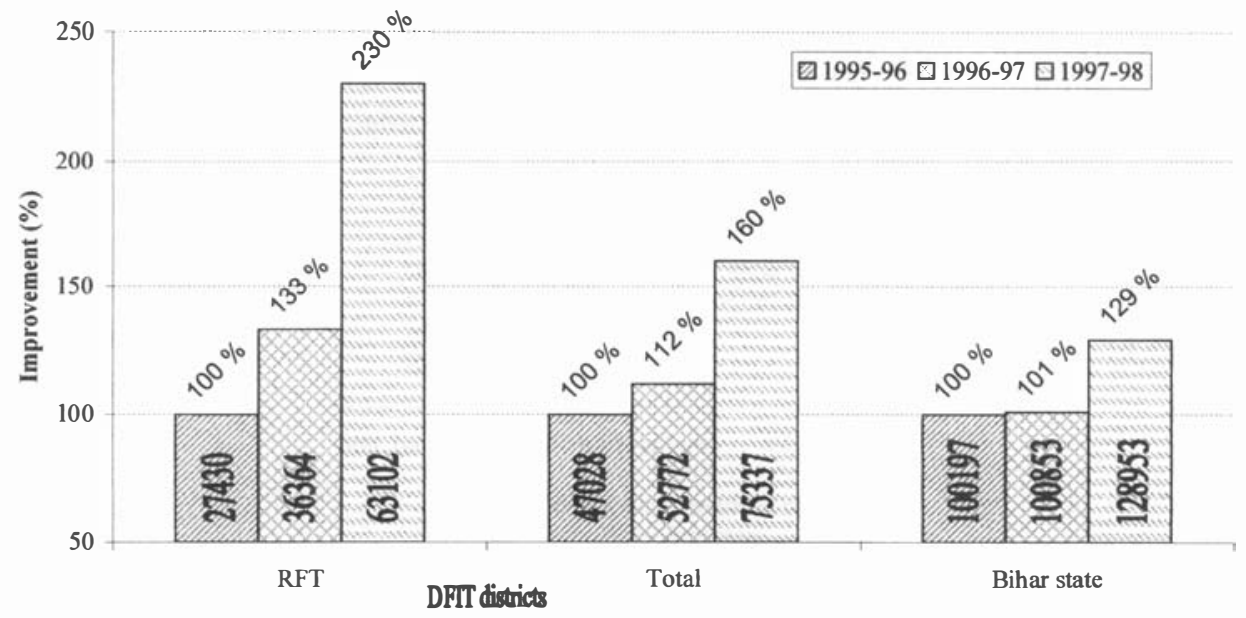

Figure 2. Case discharge. Improvement (\%) in DFIT-assisted districts and Bihar State. 


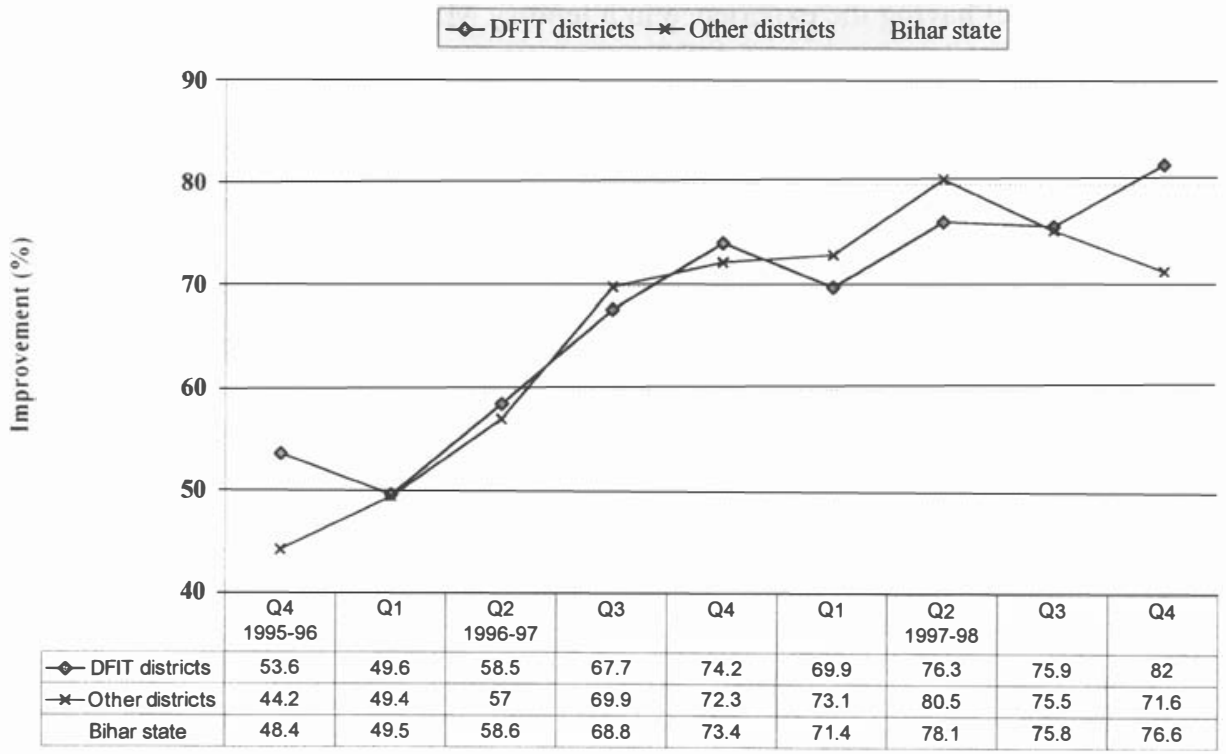

Figure 3. Recorded leprosy patients actually treated. Improvement (\%) in DFIT-assisted districts, others and Bihar State.

Though there was an improvement in the other 23 districts of Bihar State without DFIT support teams, it was marginal.

\section{Discussion}

The Leprosy Elimination Advisory Group (LEAG) (4th meeting 1998) recommended that 'The global strategy for the elimination of leprosy as a public health problem, based on implementation of MDT together with case finding activities, is continuing to prove very successful and should be pursued in all countries with increased vigour.'

'The challenge now, through an accelerated plan of action, is to bring this effective technology to all remaining hidden cases and to those living in underserved populations who have little or no access to MDT. While the progress made so far in eliminating leprosy is highly impressive, these remaining problems call for further intensification of our efforts using focused yet flexible approaches'. ${ }^{\prime}$

Bihar presented a situation where infrastructure was inadequate and staff untrained. Financial resources were available but not effectively utilized. A crude estimate of prevalent leprosy cases was about 300,000 in the state, against 127,000 active leprosy cases actually on record in 1996. Intensive screening programmes in two districts (1 week in Bhojpur district and 3 months in Singhbhum district) resulted in new case detection equivalent to the whole year's case detection. It was not possible to implement MDT programme activities (case detection, treatment delivery) as fast as in some of the other regions in India. Bihar needed technical guidance at the peripheral level to plan, implement and monitor MDT programme activities. 
DFIT personnel having the experience in a leprosy MDT programme were able to offer guidance on the implementation of MDT in an efficient manner, with concentration of technical expertise at the peripheral level, where it was mainly needed. The support teams worked with the field staff in carrying out the routine programme activities (on the job training), thus improving the morale of the leprosy workers so that they became more confident.

In any leprosy-endemic region when MDT is implemented extensively, the new case detection and cases on record are expected to increase steeply during the first 2-3 years. Decline is expected 3-5 years after implementation of the MDT programme. The DFIT support teams in 16 districts in this state were clearly able to bring about a positive change in the leprosy scenario, as shown by the remarkable improvement in new case detection, case discharge and treatment coverage. After some initial hesitation, the co-operation of programme staff was excellent, with sustained enthusiasm throughout. The experience suggests that co-operation between government and non-government staff is feasible and effective, with the potential to proceed to an elimination level.

\section{Acknowledgement}

We thank the Government of India, the State Leprosy Officer, Bihar and administrative authorities at the state level and district level for their encouragement and support. We are grateful to Dr N. B. B. Reddy for the guidance and the programme staff for their co-operation. The support teams deserve special appreciation for their patience and concerted effort. We thank the Damien Foundation India Trust for financial support.

\section{References}

${ }^{1}$ World Health Organisation. Action programme for elimination of leprosy-status report. WHO/LEP/98.2, 1998.

2 World Health Organisation. Weekly Epidemiological Record, 1998; 21.

${ }^{3}$ World Health Organisation. Weekly Epidemiological Record, 1998; 23.

${ }^{4}$ Vijayakumaran P, Reddy NBB, Krishnamurthy P, Ramanujam R. Utilising primary health care workers for case detection. Ind J Lepr, 1998; 70: 203-210.

5 Vijayakumaran P et al. Hidden cases of leprosy (in prison). Ind J Lepr, 1997; 69, 271-274. 